ARTICLE

\title{
C. elegans maximum velocity correlates with healthspan and is maintained in worms with an insulin receptor mutation
}

\author{
Jeong-Hoon Hahm ${ }^{1}$, Sunhee Kim ${ }^{1, \star}$, Race DiLoreto ${ }^{2, \star}$, Cheng Shi $^{2, \star}$, Seung-Jae V. Lee ${ }^{3}$,
} Coleen T. Murphy ${ }^{2} \&$ Hong Gil Nam ${ }^{1,4}$

Ageing is marked by physical decline. Caenorhabditis elegans is a valuable model for identifying genetic regulatory mechanisms of ageing and longevity. Here we report a simple method to assess C. elegans' maximum physical ability based on the worms' maximum movement velocity. We show maximum velocity declines with age, correlates well with longevity, accurately reports movement ability and, if measured in mid-adulthood, is predictive of maximal lifespan. Contrary to recent findings, we observe that maximum velocity of worm with mutations in daf-2(e1370) insulin/IGF-1 signalling scales with lifespan. Because of increased odorant receptor expression, daf-2(e1370) mutants prefer food over exploration, causing previous on-food motility assays to underestimate movement ability and, thus, worm health. Finally, a disease-burden analysis of published data reveals that the daf-2(e1370) mutation improves quality of life, and therefore combines lifespan extension with various signs of an increased healthspan.

\footnotetext{
${ }^{1}$ Center for Plant Aging Research, Institute for Basic Science, Daegu 42988, Republic of Korea. ${ }^{2}$ Department of Molecular Biology, Lewis-Sigler Institute for Integrative Genomics, Princeton University, 148 Carl Icahn Laboratory, Washington Road, Princeton, New Jersey 08544, USA. ${ }^{3}$ Department of Life Sciences and School of Interdisciplinary Bioscience and Bioengineering, Pohang University of Science and Technology, Pohang, Gyeongbuk 790-784, Republic of Korea. ${ }^{4}$ Department of New Biology, DGIST, Daegu 42988, Republic of Korea. ${ }^{\star}$ These authors contributed equally to this work. Correspondence and requests for materials should be addressed to C.T.M. (email: ctmurphy@Princeton.EDU) or to H.G.N. (email: nam@dgist.ac.kr).
} 
A geing is an important risk factor for disease and is accompanied by the decline of many physiological characteristics, including physical performance, negatively affecting quality of life. Therefore, quality of life with age, not just longevity, is important for our ageing society. Caenorhabditis elegans has been a valuable model organism in revealing the genetic regulatory mechanisms of ageing and longevity ${ }^{1,2}$. One of the best-studied longevity pathways is the insulin/IGF-1 signalling (IIS) pathway, which regulates longevity in response to nutrient levels through its control of the activity of the FOXO transcription factor and its downstream targets ${ }^{3-6}$. Mutations of the DAF-2 insulin receptor, particularly the canonical allele daf-2(e1370), double lifespan ${ }^{3}$ and extend the abilities to learn and remember, resist stress and infection, repair axons ${ }^{7}$, and suppress neuronal morphological defects ${ }^{8-10}$ with age. The components of the IIS pathway are well-conserved, and are linked to extreme longevity in humans ${ }^{11}$ as well. Recently, despite the fact that the daf-2 mutation extends many physiological functions with age $e^{7-10}$, Bansal et al. concluded that daf-2(e1370)
IIS mutants are less healthy than wild-type animals, disproportionately extending their 'unhealthy' lifetime ${ }^{2,12}$.

One of the most powerful tests of physical ability in elderly humans is the short physical performance battery $(\mathrm{SPPB})^{13}$, an assessment of the maximum exercise capacity of lower extremities over a short period of time. The SPPB accurately predicts risk of disability ${ }^{14}$, length of hospital stay, and mortality over the year after hospital discharge ${ }^{15}$. The SPPB includes a short $(4 \mathrm{~m})$ walk to measure maximum gait speed. Like humans, $C$. elegans shows age-related decline in physical ability, which is manifested by reduced body movement ${ }^{16-22}$. We wondered whether a similar metric of maximum velocity (MV) in a short period (30 s) would be equally informative in C. elegans. We found that worms' MV declines with age, correlates well with longevity, accurately reports movement ability, and importantly, is predictive of future longevity. daf-2(e1370) mutations extend MV and 'healthspan', scaling with lifespan ${ }^{19}$. Motility assays performed on bacteria underestimate daf-2(e1370) mutants' movement ability due to their high odr-10 levels and subsequent preference for food over

a
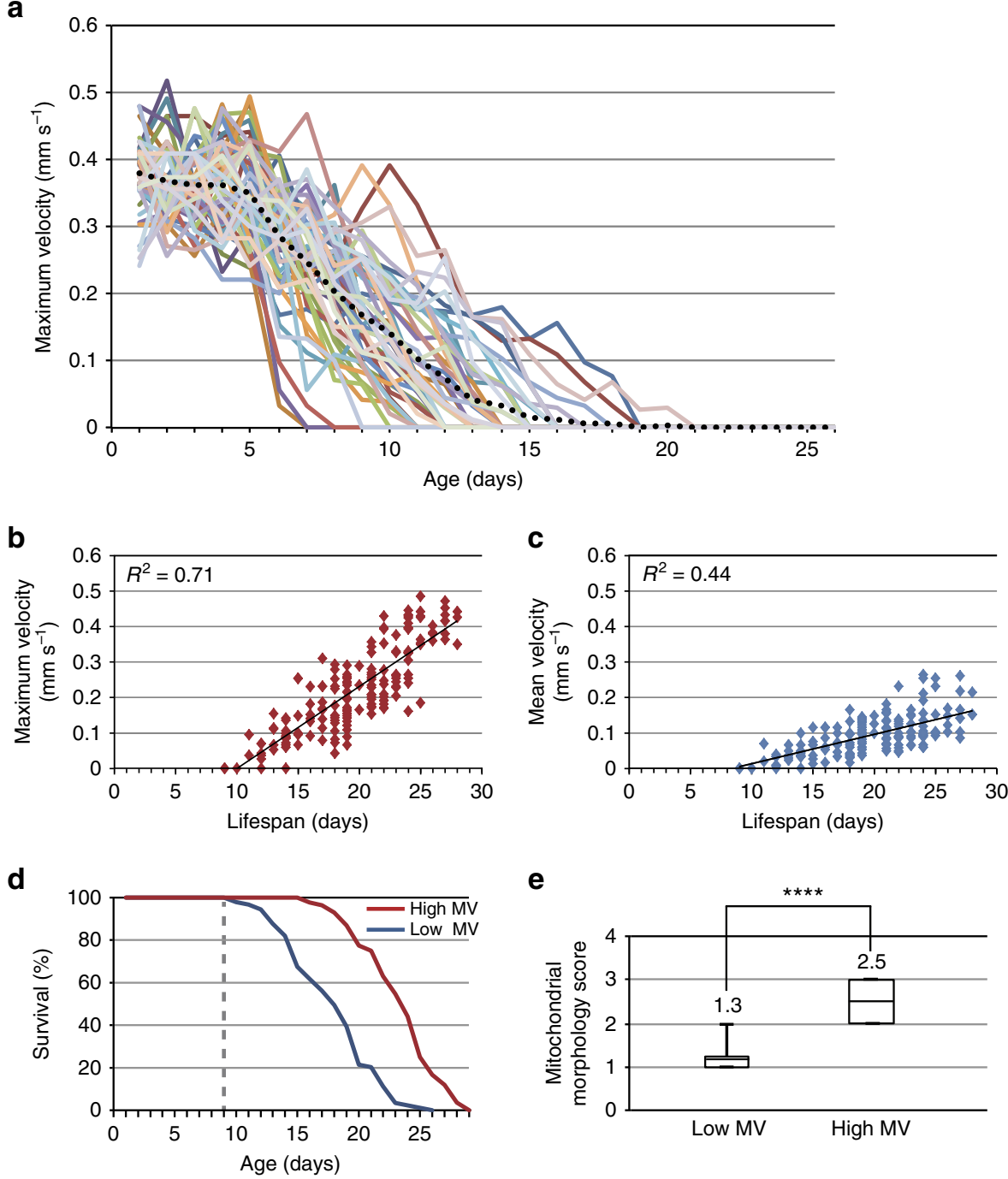

Figure 1 | MV is correlated with longevity. (a) Age-dependent changes in the MV of 52 individual wild-type worms. Black dots represent the average value at each age. $(\mathbf{b}, \mathbf{c})$ Correlation between MV and lifespan (b) and correlation between mean velocity and lifespan (c) of individual worms. The MVs and mean velocities of individual worms were measured at day 9 of adulthood and their lifespans were measured thereafter. $r^{2}$, coefficient of determination. $n=173$. (d) Survival curves of the low-MV $(n=89)$ and high-MV $(n=84)$ group worms. The groups were divided at day 9 of adulthood (grey dotted line) and the survivorship of worms in each group was measured thereafter. (e) Correlation of MV with mitochondrial morphology at day 9 of adulthood. Scores of mitochondrial morphology of low-MV and high-MV groups were $1.3 \pm 0.5$ and $2.5 \pm 0.5$, respectively $(n=27)$. The bottom and top of the box and whiskers are the first and third quartiles, and the band inside the box is the average. Significance was determined using a two-tailed, unpaired $t$-test. ${ }^{\star \star \star \star} P<0.0001$. 
exploration. We used human disease-burden models to assess C. elegans mutants' quality of life; quality-adjusted reanalysis of data from previously published healthspan studies ${ }^{2,19}$ demonstrates that daf-2(e1370) mutants' healthspan correlates with longevity extension, and in terms of disease-burden analysis, improves quality of life. The IIS pathway mutation daf-2(e1370) coordinates the extension of lifespan with many aspects of healthspan, providing valuable insight into mechanisms that extend functional health with age.

\section{Results}

MV correlates with lifespan. To mimic the SPPB test used to assess health in elderly humans, we measured worms' MV over $30 \mathrm{~s}$ on an unseeded nematode growth medium (NGM) plate each day of adulthood (Supplementary Fig. 1). Our assay differs from previous motility assays in its brevity (30 s versus 5 min (ref. 21) or longer ${ }^{23}$ ), longitudinal testing of individual worms rather than populations, and measurement on plates without bacteria ${ }^{2}$.MV appears to not simply be a response to harsh touch, as harshtouch mutants behave like wild-type worms in the assay, and the MV is distributed over the entire $30 \mathrm{~s}$ window for all animals (Supplementary Fig. 2).

To assess age-associated decline of physical performance, we measured MV of 52 individual wild-type worms each day of their lifespan (Fig. 1a). We found that motility decreased with age (Supplementary Fig. 3), with MV declining earlier and more drastically than mean velocity (Supplementary Fig. 3). During the early phase of adulthood (day 1-5), MV of individual worms was maintained in the range of $0.22-0.52 \mathrm{~mm} \mathrm{~s}^{-1}$. However, from day 6 onwards, all worms showed a decline in MV with age.

We grouped worms into high $\left(\geq 0.22 \mathrm{~mm} \mathrm{~s}^{-1}\right)$ and low $\left(\leq 0.21 \mathrm{~mm} \mathrm{~s}^{-1}\right) \mathrm{MVs}$; the criterion was based on the minimum MV at day 1 of adulthood. The population of the low-MV group increased almost exponentially, reaching $54.6 \%$ by day 9 of adulthood (Supplementary Fig. 4). We then examined the correlation between MV of worms at day 9 of adulthood (midlife) and their lifespan thereafter. Day 9 MV correlated well with lifespan (coefficient of determination $\left(r^{2}\right)=0.71$ ), better than the correlation between lifespan and mean velocity $\left(r^{2}=0.44\right.$; Fig. $\left.1 \mathrm{~b}, \mathrm{c}\right)$, thrashing rate $\left(r^{2}=0.18\right.$; Supplementary Fig. 5), or pharyngeal pumping $\left(r^{2}=0.65\right.$; Supplementary Fig. 5). Remarkably, the median lifespan of worms in the day 9 high-MV group ( $23 \pm 3.2$ days) was $35.3 \%$ longer than that of the worms in the day 9 low-MV group $(17 \pm 3.6$ days; Fig. 1d). Thus, MV of wild-type worms at day 9 of adulthood is a reliable predictor of longevity thereafter.

In humans, mitochondrial defects are associated with ageing of skeletal muscle and are correlated with reduced physical strength in the elderly ${ }^{24-26}$. Young C. elegans' mitochondria are tubular ${ }^{27}$; however, gradual fragmentation with age results in sparse, globular mitochondria (Supplementary Fig. 6). At day 9 of adulthood, worms in the low-MV range had very fragmented mitochondria, while the high-MV range group's mitochondria were significantly less damaged at the same age (Fig. le and Supplementary Fig. 7). In addition, expression of the protective lifespan biomarker sod-3 (ref. 28) was 1.7-fold higher in the highMV group than in the low-MV group (Supplementary Fig. 8).

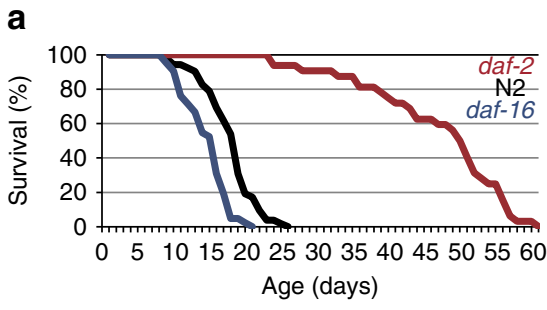

C

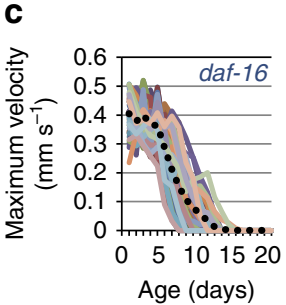

d
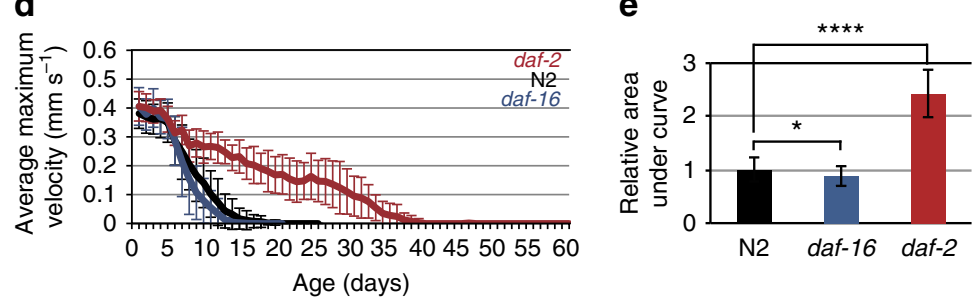

f

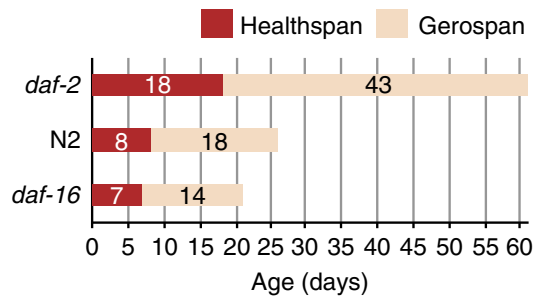

b

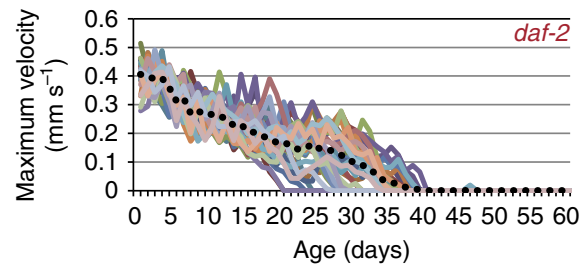

e

g

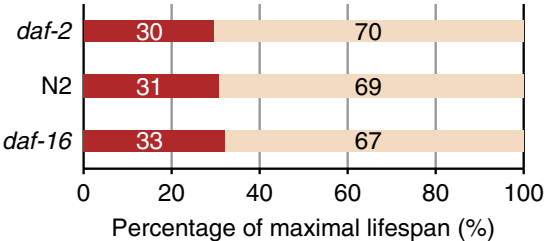

Figure 2 | Correlation of $\mathbf{M V}$ with lifespan in daf-2 mutants. (a) Age-associated change of survivorship in N2 (wild type) ( $n=52)$, daf-2(e1370) ( $n=32)$ and daf-16(mu86) $(n=42)$ worms. (b,c) Age-associated change of MVs in daf-2 (b) and daf-16 mutant worms (c). (d) Age-associated change of average MVs in N2 $(n=52)$, daf-2 $(n=32)$ and daf-16 $(n=42)$ mutant worms. Error bars represent s.d. (e) A cumulative difference in physical performance in N2 and daf- 16 and daf- 2 mutant worms as indicated by area under curves in $\mathbf{d}$. The relative values of area under curves are 1.0 $\pm 0.2,0.9 \pm 0.2$ and $2.4 \pm 0.4$ for N2, daf-16 and daf-2 worms, respectively. (f) Comparison of healthspan and gerospan in N2, daf-16 and daf-2 worms. Healthspan was defined as 'the period during which the worm showed $>50 \%$ maximal functional capacity of wild type'2. Gerospan was defined as 'the period during which the worm showed $<50 \%$ maximal functional capacity of wild type' ${ }^{2}$. (g) The healthspan-to-gerospan ratio normalized to their maximal lifespan in N2, daf-16 and daf-2 worms. 
These data show that MV correlates with two age-associated physiological parameters, and suggest that mechanisms determining MV might also regulate mitochondrial integrity maintenance and stress protection gene expression.

daf-2(e1370) mutation extends healthspan. We next assessed the MV of long-lived daf-2(e1370) insulin/IGF-1 receptor mutants and short-lived mutants of the DAF-16 FOXO transcription factor (daf-16(mu86)), which is required for daf-2 mutants' longevity $^{3,4,29}$ (Fig. 2a). daf-2 animals exhibited a higher MV with age, particularly from day 10 onwards (Fig. 2b-d). At day 26 of adulthood, when all the wild-type worms had died, daf- 2 mutants still maintained on average $36 \%$ of MV (Fig. 2d). We examined the correlation of MV on each day with the lifespan for the worms in the longitudinally tracked cohort (Figs $1 \mathrm{a}$ and 2b,c). The correlation between $\mathrm{MV}$ and lifespan is best on day 9 for $\mathrm{N} 2$ worms, day 23 for daf-2 and day 10 for daf-16 (Supplementary Fig. 9); in all cases, the best correlation between MV and lifespan occurs within 1 day of the first death in the cohort. We integrated the area under the MV curve to serve as an indicator of overall physical performance; daf-2 mutants showed a 2.4 -fold increase over wild type, and daf-16 mutants showed slightly lower physical performance (Fig. 2e). Thus, daf-2(e1370) mutation extends physical ability with age.

We then used MV to determine the 'healthspan' (defined as the period with $>50 \%$ of maximal activity ${ }^{2}$ ) and 'gerospan' (period with $<50 \%$ maximal activity ${ }^{2}$ ) ratios of wild-type, daf-16(mu86), and daf-2(e1370) mutant worms, in the same manner as Bansal et al. ${ }^{2}$. While this type of analysis does not include any error calculation, our results suggest that daf-2 mutants increased healthspan more than twofold compared with wild-type and daf-16 mutants (Fig. 2f), and the resulting normalized healthspan-togerospan ratios were similar among the three strains (Fig. 2g). Thus, daf-2(e1370) mutation extends both lifespan and healthspan to a similar extent, without proportionally extending the unhealthy part of life, in contrast to Bansal et al. ${ }^{2}$ conclusions.

odr-10 levels determine on-food motility rates. We were concerned that differences in motility assays may have led to discrepancies with the previous report ${ }^{2}$. Our assay measures MV on plates without bacteria, while Bansal et al. ${ }^{2}$ measured the distance worms moved on bacteria. We wondered whether daf-2's lower motility on food might not reflect its ability to move, but rather its preference for food over exploration. Food-seeking preference is determined by levels of the odorant receptor odr-10 (ref. 30); high levels of odr-10 suppress food-exploration behaviour ${ }^{30}$. Ryan et al. ${ }^{30}$ showed that odr-10, despite the fact that it is only expressed in the AWA neurons and primarily detects diacetyl, plays a major role in food detection and is the primary factor in determining the decision of males to explore (mate search) rather than feed. Indeed, we found that the expression of odr-10, which contains a DAF-16-binding element $809 \mathrm{bp}$ upstream of its start site, is elevated 15-20-fold in daf-2(e1370) mutants (Fig. 3a). Furthermore, we observed that reduction of odr-10 levels dramatically increases daf-2's motility on bacteria: unlike control-treated animals, which stop moving after 1.5-3 min when placed onto bacteria, daf-2(e1370);odr-10(RNAi) worms continue to move constantly over a $10 \mathrm{~min}$ assay period (Fig. $3 \mathrm{~b}$ ), and reduction of wild-type worms' $o d r-10$ levels has no significant effect (Fig. 3c and Supplementary Movies 1-3). (Interestingly, knockdown of other genes involved in odour detection, including $o d r-3, o d r-7$ and osm-9, did not have the same effect on daf-2(e1370) motility (Supplementary Fig. 10).) Therefore, motility can only be measured accurately off food, because movement on bacteria measures food-seeking rather than ability to move.
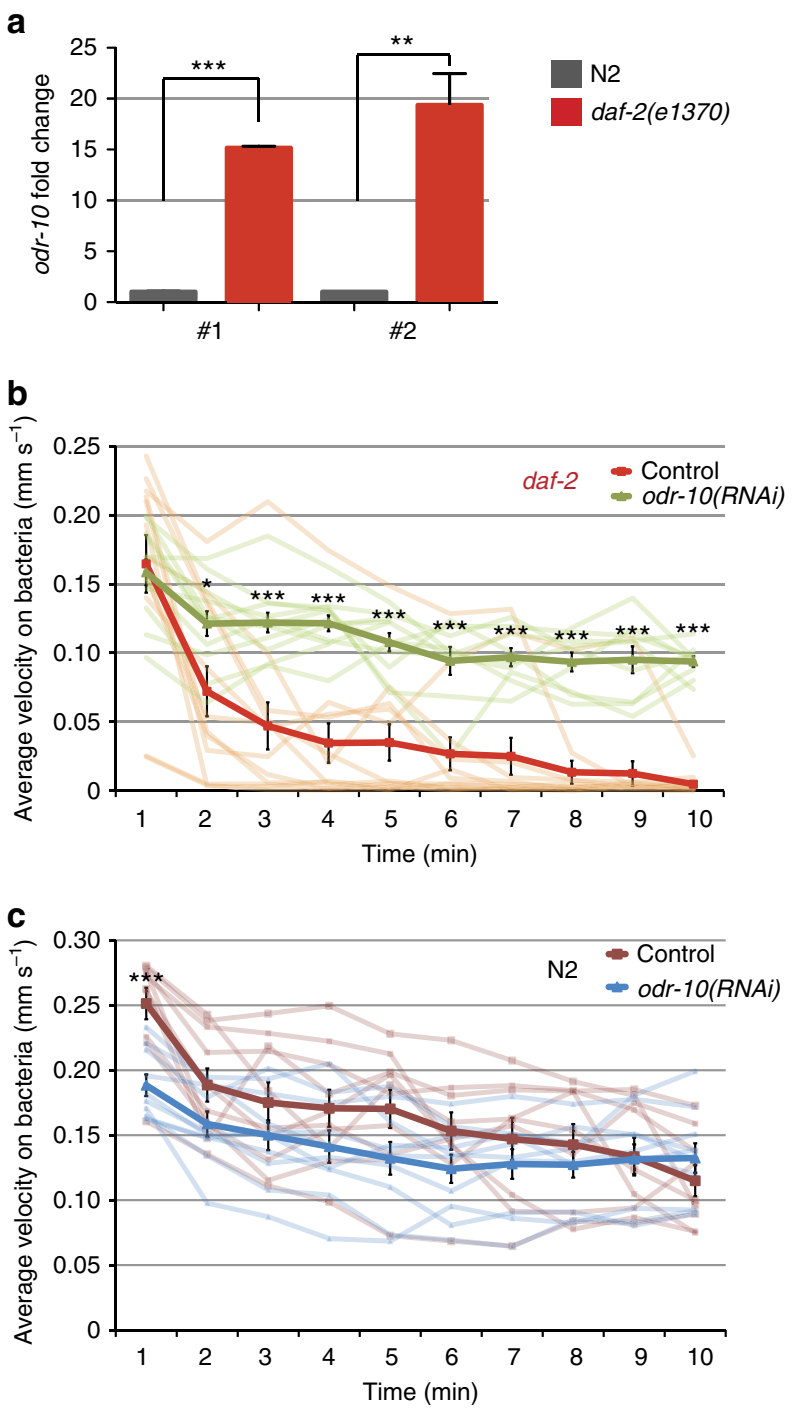

Figure 3 | Motility on bacteria measures food preference rather than ability to move. (a) Two sets of primers (\#1 and \#2) were used to measure the expression of odr-10 by qRT-PCR in N2 and daf-2 mutants. For each primer set, odr-10 expression in wild type was normalized to 1 . odr-10 expression in daf- 2 mutants increased by $15.2 \pm 0.2$-fold and $19.4 \pm 3.1$-fold, respectively. (b) Reduction of odr-10 levels by RNAi increased daf-2 motility on bacteria significantly. daf-2 on OP50: $0.04 \pm 0.03 \mathrm{~mm} \mathrm{~s}^{-1}$; daf-2; odr-10(RNAi): $0.11 \pm 0.01 \mathrm{~mm} \mathrm{~s}^{-1}$ (c) wild-type worms' motility on bacteria was not significantly affected by reduction of odr-10: N2 worms on OP50: $0.17 \pm 0.03 \mathrm{~mm} \mathrm{~s}^{-1}$; N2 on odr-10(RNAi): $0.14 \pm 0.03 \mathrm{~mm} \mathrm{~s}^{-1} \cdot n=3$, ${ }^{\star} P<0.05$, ${ }^{\star \star} P<0.01,{ }^{\star \star \star} P<0.001,{ }^{\star \star \star \star} P<0.0001$; unpaired $t$-test.

daf-2(e1370) mutation increases quality of life. As MV is only one metric of healthspan, we reanalysed the entire Bansal et al. ${ }^{2}$ healthspan data set. (The same daf-2 allele, e1370, was used in their studies, as well.) In our analysis, we normalized each health metric at each time point to the maximum score of wild type to directly compare performance levels (Fig. 4a). This reanalysis shows that daf-2 mutants were healthier than wild type in all metrics except thrashing in liquid. However, previous thrashing measurements of daf-2 mutants ${ }^{31}$ and another long-lived IIS mutant, age-1 (ref. 32), demonstrate that these IIS mutants maintain thrashing activity with age better than wild type.

We wondered whether cost-utility (disease burden) methods developed to assess quality of life ${ }^{33}$ (which are typically used to make cost assessments of human disease treatment options) 
a

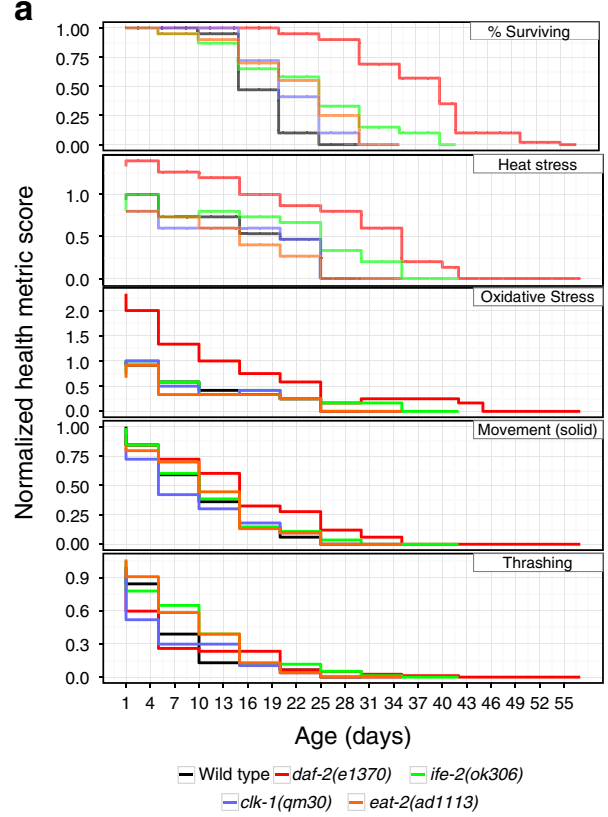

C

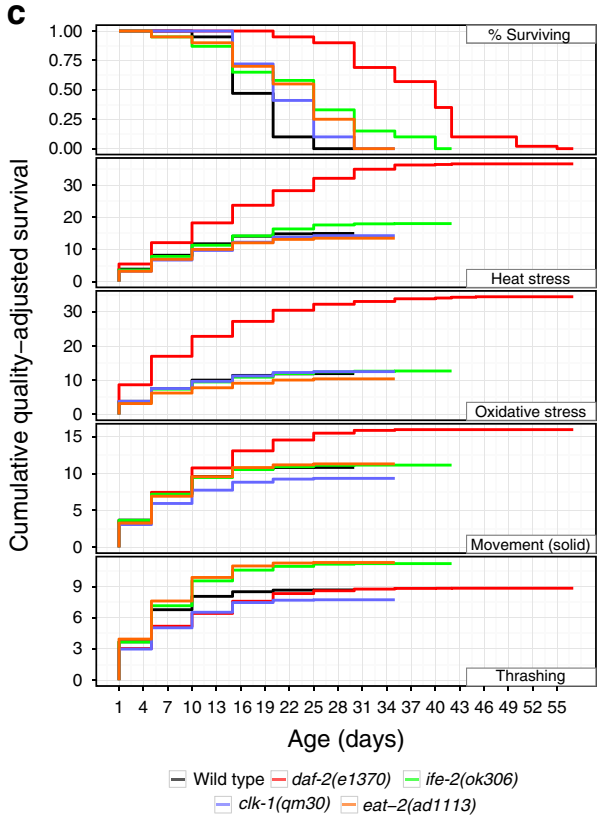

b

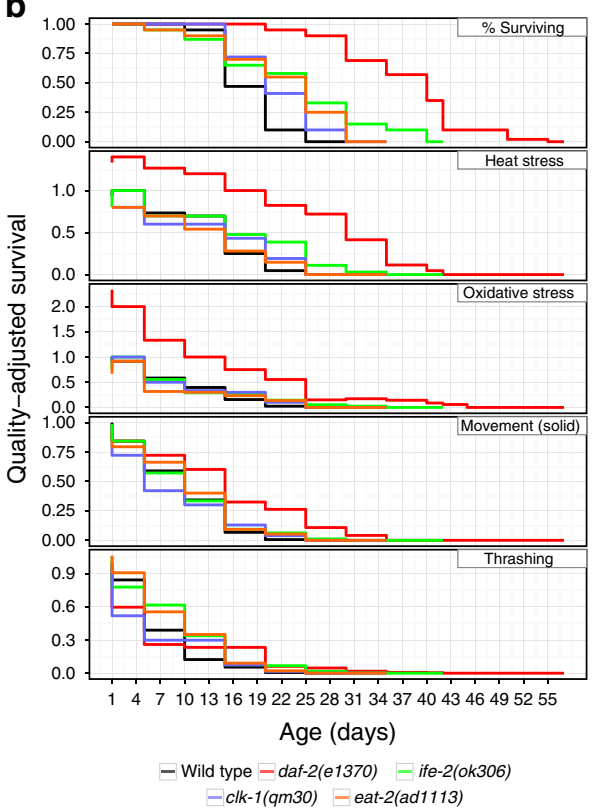

d

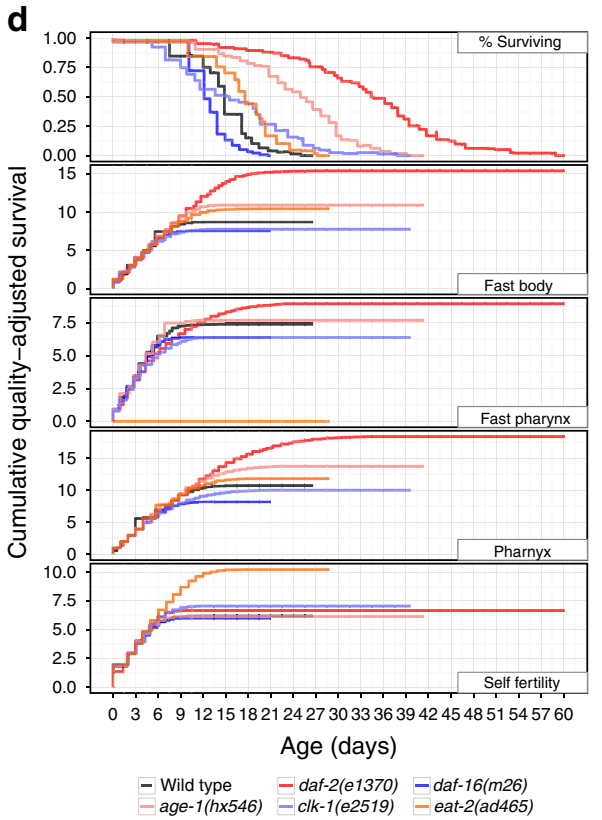

Figure 4 | Quality-adjusted health metrics. daf-2 mutants are healthier than wild type. (a) Quality-adjusted lifespan curves for the health metrics observed by Bansal et al. ${ }^{2}$, with each metric normalized to the maximum value of the wild type. (b) The quality-adjusted lifespan curve is the survival rate multiplied by the normalized health measurement. (c) Visualizing the cumulative area under the quality-adjusted lifespan curve at each time point shows that daf- 2 mutants have higher lifespan quality over most measured health metrics. (d) Analysis of additional healthspan data measured by Huang et al. ${ }^{19}$ shows a similar increase in total lifespan quality of daf-2 mutants over wild type.

could be applied to these healthspan analyses of C. elegans. To do so, we combined information from the lifespan curve and health measurements reported by Bansal et al. to obtain a 'quality-adjusted lifespan' curve, according to the method of Zeckhauser and Shepard ${ }^{33}$. We multiplied the lifespan curve by the normalized healthspan curve, producing a curve that describes both the declining health of the animal and the declining survival of the population (Fig. 4b). By calculating the area under the curve (AUC) of the quality-adjusted lifespan, we can visualize total quality of life, taking into account both survival and health (Fig. 4c; Methods section). Reanalysis of the Bansal et al. ${ }^{2}$ data using this approach suggests that while not all longevity mutants have a commensurate increase in life quality (in fact, some longevity mutants, such as clk-1, exhibit lower total quality, as Bansal et al. reported), increased health and extended lifespan result in a higher total quality for daf-2(e1370) mutants. (We note that the cumulative quality-adjusted score for thrashing is equivalent for daf-2(e1370) and wild type, but that thrashing has little correlation overall with longevity (Supplementary Fig. 5).) We reached a similar conclusion upon reanalysis of the data from Huang et al. ${ }^{19}$, which also measured several healthspan parameters (Fig. $4 \mathrm{~d}$ and Supplementary Fig. 11a,b). In addition, applying this analysis to our own MV data shows the same dramatic increase in daf-2(e1370) lifespan quality (Supplementary 
Fig. 11c-e). Thus it appears from multiple analyses of healthspan that daf-2(e1370) mutants have a higher quality of life than wildtype worms.

\section{Discussion}

Here we have modelled the SPPB assessment of physical ability in elderly humans in worms, using a simple assay of C. elegans' maximum ability to move over a short period. MV correlates well with age; in fact, MV performance at midlife (day 9) can predict the remaining lifespan of the individual. Our analysis also reveals that other widely used healthspan metrics are less correlated with lifespan; thrashing is a particularly poor correlate $\left(r^{2}=0.18\right)$, while pumping has a good correlation $\left(r^{2}=0.65\right)$ but shows highly irregular patterns (Supplementary Fig. 5). Thus, MV is a powerful healthspan proxy and may be the most informative metric of C. elegans' health with age.

An unexpected benefit of this experimental design is that it is not confounded by the worms' ongoing assessment of its bacterial food source. We discovered that daf-2 has an inherently higher preference for food than exploration, likely due to its increased expression level of the daf-2/daf-16 target odr-10; high levels of the ODR-10 odour receptor cause daf-2 worms to prefer food over exploration, slowing its movement on bacteria. (Further, our data on other chemosensory genes suggest that odr-10 is a particularly good indicator of food preference.) As this type of movement is not limited by ability, but rather by preference, it may be difficult to draw conclusions regarding healthspan from on-food motility assays.

Measurements that take into account both lifespan and healthiness are important in assessing quality of life with age, and the clinical implications of coordinated extension of lifespan and healthspan are immense. Not all lifespan interventions will improve health or total quality, as $c l k-1$ mutants demonstrate. In fact, several longevity mutants are less healthy overall than wild-type worms by different measurements, and it is important to note which mutants may have a long life with no health benefit. However, we find no evidence to support the claim by Bansal et al. that daf-2(e1370) mutants spend a greater fraction of life in a frail state; in fact, daf-2 mutants demonstrate that healthspan and lifespan can be coordinately extended. From the analysis of our own data and that of published experiments, it seems clear that daf-2 extends not only lifespan, but also many abilities with age. In addition to the standard $C$. elegans-specific 'healthspan' assays (heat stress, oxidative stress and movement), daf-2 extends behaviours that are relevant for human decline with age, as well. This includes the abilities to learn and remember ${ }^{34}$, repair axons ${ }^{7}$, resist pathogenic infections ${ }^{35}$, suppress age-related neuronal morphological defects ${ }^{8-10}$ and neurodegenerative protein aggregation ${ }^{36}$, maintain neuromuscular junction activity ${ }^{23,37}$, and maintain high-quality oocytes with age ${ }^{38}$. Many of the downstream targets of the daf-2 insulin signalling pathway $^{6}$ that enable these functions have been identified, and many of these genes and the underlying mechanisms are conserved in mammals; therefore, the extended abilities that daf-2 exhibits offers therapeutic target possibilities.

Defining 'healthspan' and 'gerospan' based on population maximum lifespans ignores the fact that quality is a continuum. Instead, our analysis takes into account the length of time an individual can expect to live, and how healthy that individual can expect to be with age. In the case of daf-2(e1370) mutants, quality of life is clearly superior. The mechanistic study of coordinated health and life extension might allow the development of therapeutics to remedy end-of-life problems or to compress morbidity, decreasing health costs. C. elegans IIS longevity mutants remain valuable tools in understanding the mechanisms by which we might achieve these goals.

\section{Methods}

Strains. The C. elegans strain, N2 Bristol, was used as the wild type. The N2 strain and the insulin/IGF-1 mutants, daf-2(e1370), daf-16(mu86), CF1553 muIs84[pAD76 (sod-3::GFP)] and PD4251 ccIs4251 I; dpy-20(e1282) IV were obtained from the Caenorhabditis Genetics Center.

Measurement of worm MV. Synchronized worms were prepared by placing adult worms on a NGM plate and allowing them to lay eggs for $3 \mathrm{~h}$. After removing the adult worms, each synchronized progeny was transferred to a single NGM plate and cultured to L4 stage. After $24 \mathrm{~h}$, single worms were transferred to the physical assay plate and movements were recorded immediately. The assay conditions were as follows: $20^{\circ} \mathrm{C}$ and $\sim 40 \%$ humidity, with no lid. The physical assay plate was prepared in the same manner as the NGM, but with no bacterial lawn. The recording system comprised a stereomicroscope (Nikon SMZ 745T), a chargecoupled device camera (TUCSEN TCH-5.0) and imaging software (TUCSEN ISCapture). A $\times 12.14$ zoom lens was used to keep the worms within the field of view. Movement was recorded every $24 \mathrm{~h}$ throughout the lifespan of each worm. The recording period was $30 \mathrm{~s}$ at a rate of 30 frames per second. After recording, the worm was transferred to a fresh NGM plate (the 'growth plate' shown in Supplementary Fig. 1). The locomotion velocity was expressed as $\mathrm{mm}$ per second (the distance ( $\mathrm{mm}$ ) between displaced centroids per second). Recorded images were analysed by ImageJ and wrMTrck (plugin for ImageJ: www.phage.dk/plugins). The locomotion velocity data were imported into an Excel spreadsheet. The peak locomotion velocity observed in the $30 \mathrm{~s}$ period was used as the MV.

Lifespan analysis. Lifespan was assessed on NGM plates at $20^{\circ} \mathrm{C}$. The number of live animals was scored every day until death. Lifespan was analysed by Oasis survival analysis software ${ }^{39}$.

Pharyngeal pumping and thrashing rate decline assays. Synchronized single worms were observed every day. The number of contractions in the terminal bulb of pharynx was counted for $30 \mathrm{~s}(n=19)$. The change in the reciprocating motion of bending at the mid-body in S-basal medium was counted as a body bend. The number of body bends was counted for $30 \mathrm{~s}(n=20)$.

Qualitative analysis of mitochondrial morphology. Morphological categories were defined according to Regmi et al. ${ }^{27}$ : (1) images showing a majority of long interconnected mitochondrial networks were classified as tubular; (2) images showing a combination of interconnected mitochondrial networks along with some smaller fragmented mitochondria were classified as intermediate; (3) images showing a majority of short mitochondria were classified as fragmented; and (4) images showing sparse globular mitochondria were classified as very fragmented. We scored mitochondrial morphology as '1' (very fragmented), '2' (fragmented), ' 3 ' (intermediate) and '4' (tubular; Supplementary Fig. 7). Mitochondrial morphology was examined in PD4251 strains at day 9 of adulthood after MV was recorded. Worms were immobilized during imaging using $30 \mathrm{mM}$ sodium azide. Imaging was performed using a microscope equipped with a C-Apochromat $40 \mathrm{x} / 1.20 \mathrm{~W}$ Korr FCS M27 and a photo-multiplier tube. Zen 2011software (black edition) was used to acquire fluorescent $\mathrm{z}$ stacks of individual animals $(1 \mu \mathrm{m}$ per slice).

Observation of sod-3 expression. sod-3 expression was examined in CF1553 strain worms at day 9 of adulthood after MV was recorded. Imaging was performed using a microscope equipped with a C-Apochromat 10x/1.20W Korr FCS M27 and a photo-multiplier tube. Zen 2011 software (black edition) was used to acquire fluorescent $\mathrm{z}$ stacks of individual animals ( $1 \mu \mathrm{m}$ per slice). Worms were immobilized during imaging using $30 \mathrm{mM}$ sodium azide.

odr-10 qRT-PCR measurements. Two sets of primers (\#1 and \#2) were used to measure the expression of $o d r-10$ by quantitative reverse transcription PCR (qRT-PCR) in N2 and daf-2 mutants. For each primer set, odr-10 expression in wild type was normalized to $1 . p m p-3$ was used as a reference gene.

'odr-10-qRT-1-r' 5'-AACGGTGCGATGAACATGAC-3'

'odr-10-qRT-1-f 5' -CTTCAGGTATCCCGATCTGAAACT-3'

'odr-10-qRT-2-r' 5'-TCAGATCGGGATACCTGAAGA-3'

'odr-10-qRT-2-f 5'-TCTACTGCGGATATGCCACG-3'

Reference gene primers: $p m p-3$

Forward: $5^{\prime}$-AGTTCCGGTTGGATTGGTCC- $3^{\prime}$

Reverse: $5^{\prime}$-CCAGCACGATAGAAGGCGAT-3'

Quality-adjusted lifespan analysis. Quality metrics were taken from published work. Because the raw data from Bansal et al. ${ }^{2}$ were not made publicly available by the authors at time of submission of this paper, we extracted data from their figures using GetData Graph Digitizer. (No N or error information is available, however.) The lifespan $(\mathrm{L}(\mathrm{t}))$ (\% surviving with age) and healthspan $(\mathrm{H}(\mathrm{t}))$ (health measurement with age) data were graphed using a left-hand step function. The curves were combined by multiplying the lifespan and healthspan curves together, with the value of the resulting quality-adjusted lifespan curve $(\mathrm{Q}(\mathrm{t}))$ being 
$\mathrm{Q}(\mathrm{t})=\mathrm{L}(\mathrm{t}) \times \mathrm{H}(\mathrm{t})$. The AUCs was calculated by taking the definite integral of this step function. The set of timepoints $\mathrm{T}$ is zero-indexed and consists of $\mathrm{T}=\left\{\mathrm{t}_{0}, \mathrm{t}_{1}, \ldots, \mathrm{t}_{\mathrm{n}}\right\}$, and we calculate the AUC as shown in equation (1).

$$
\mathrm{AUC}=\sum_{i=1}^{n} Q\left(t_{i}\right) \times\left(t_{i}-t_{i-1}\right)
$$

\section{References}

1. Kenyon, C. J. The genetics of ageing. Nature 464, 504-512 (2010).

2. Bansal, A., Zhu, L. H. J., Yen, K. \& Tissenbaum, H. A. Uncoupling lifespan and healthspan in Caenorhabditis elegans longevity mutants. Proc. Natl Acad. Sci. USA 112, E277-E286 (2015).

3. Kenyon, C., Chang, J., Gensch, E., Rudner, A. \& Tabtiang, R. A C. elegans mutant that lives twice as long as wild type. Nature 366, 461-464 (1993).

4. Ogg, S. et al. The Fork head transcription factor DAF-16 transduces insulin-like metabolic and longevity signals in C-elegans. Nature 389, 994-999 (1997).

5. Lin, K., Hsin, H., Libina, N. \& Kenyon, C. Regulation of the Caenorhabditis elegans longevity protein DAF-16 by insulin/IGF-1 and germline signaling. Nat. Genet. 28, 139-145 (2001).

6. Murphy, C. T. et al. Genes that act downstream of DAF-16 to influence the lifespan of Caenorhabditis elegans. Nature 424, 277-283 (2003).

7. Byrne, A. B. et al. Insulin/IGF1 signaling inhibits age-dependent axon regeneration. Neuron 81, 561-573 (2014).

8. Toth, M. L. et al. Neurite sprouting and synapse deterioration in the aging Caenorhabditis elegans nervous system. J. Neurosci. 32, 8778-8790 (2012).

9. Tank, E. M., Rodgers, K. E. \& Kenyon, C. Spontaneous age-related neurite branching in Caenorhabditis elegans. J. Neurosci. 31, 9279-9288 (2011).

10. Pan, C. L., Peng, C. Y., Chen, C. H. \& McIntire, S. Genetic analysis of agedependent defects of the Caenorhabditis elegans touch receptor neurons. Proc. Natl Acad. Sci. USA 108, 9274-9279 (2011).

11. Barzilai, N. et al. Genetic studies reveal the role of the endocrine and metabolic systems in aging. J. Clin. Endocrinol. Metab. 95, 4493-4500 (2010).

12. Bansal, A. \& Tissenbaum, H. A. Quantity or Quality? The Scientist (2015).

13. Guralnik, J. M. et al. A short physical performance battery assessing lower extremity function: association with self-reported disability and prediction of mortality and nursing home admission. J. Gerontol. 49, M85-M94 (1994).

14. Studenski, S. et al. Physical performance measures in the clinical setting. J. Am. Geriatr. Soc. 51, 314-322 (2003)

15. Volpato, S. et al. Predictive value of the short physical performance battery following hospitalization in older patients. J. Gerontol. A Biol. Sci. Med. Sci. 66, 89-96 (2011).

16. Croll, N. A., Smith, J. \& Zuckerman, B. M. The aging process of the nematode Caenorhabditis elegans in bacterial and axenic culture. Exp. Aging Res. 3, 175-199 (1977).

17. Duhon, S. A. \& Johnson, T. E. Movement as an index of vitality - comparing wild-type and the age-1 mutant of Caenorhabditis elegans. J. Gerontol. A Biol. Sci. Med. Sci. 50, B254-B261 (1995).

18. Herndon, L. A. et al. Stochastic and genetic factors influence tissue-specific decline in ageing C. elegans. Nature 419, 808-814 (2002).

19. Huang, C., Xiong, C. J. \& Kornfeld, K. Measurements of age-related changes of physiological processes that predict lifespan of Caenorhabditis elegans. Proc. Natl Acad. Sci. USA 101, 8084-8089 (2004).

20. Glenn, C. F. et al. Behavioral deficits during early stages of aging in Caenorhabditis elegans result from locomotory deficits possibly linked to muscle frailty. J. Gerontol. A Biol. Sci. Med. Sci. 59, 1251-1260 (2004).

21. Hsu, A. L., Feng, Z. Y., Hsieh, M. Y. \& Xu, X. Z. S. Identification by machine vision of the rate of motor activity decline as a lifespan predictor in C. elegans. Neurobiol. Aging 30, 1498-1503 (2009).

22. Garigan, D. et al. Genetic analysis of tissue aging in Caenorhabditis elegans: a role for heat-shock factor and bacterial proliferation. Genetics 161, 1101-1112 (2002).

23. Liu, J. et al. Functional aging in the nervous system contributes to age-dependent motor activity decline in C. elegans. Cell Metab. 18, 392-402 (2013).

24. Crane, J. D., Devries, M. C., Safdar, A., Hamadeh, M. J. \& Tarnopolsky, M. A. The effect of aging on human skeletal muscle mitochondrial and intramyocellular lipid ultrastructure. J. Gerontol. A Biol. Sci. Med. Sci. 65, 119-128 (2010)

25. Johnson, M. L., Robinson, M. M. \& Nair, K. S. Skeletal muscle aging and the mitochondrion. Trends Endocrinol. Metab. 24, 247-256 (2013).

26. Marzetti, E. et al. Mitochondrial dysfunction and sarcopenia of aging: from signaling pathways to clinical trials. Int. J. Biochem. Cell Biol. 45, 2288-2301 (2013).
27. Regmi, S. G., Rolland, S. G. \& Conradt, B. Age-dependent changes in mitochondrial morphology and volume are not predictors of lifespan. Aging 6, 118-130 (2014).

28. Sánchez-Blanco, A. \& Kim, S. K. Variable pathogenicity determines individual lifespan in Caenorhabditis elegans. PLoS Genet. 7, e1002047 (2011).

29. Lin, K., Dorman, J. B., Rodan, A. \& Kenyon, C. daf-16: An HNF-3/forkhead family member that can function to double the lifespan of Caenorhabditis elegans. Science 278, 1319-1322 (1997).

30. Ryan, D. A. et al. Sex, age, and hunger regulate behavioral prioritization through dynamic modulation of chemoreceptor expression. Curr. Biol. 24, 2509-2517 (2014).

31. Samuelson, A. V., Carr, C. E. \& Ruvkun, G. Gene activities that mediate increased lifespan of C. elegans insulin-like signaling mutants. Genes Dev. 21, 2976-2994 (2007).

32. Restif, C. et al. CeleST: computer vision software for quantitative analysis of C. elegans swim behavior reveals novel features of locomotion. PLoS Comput. Biol. 10, 12 (2014).

33. Zeckhauser, R. \& Shepard, D. Where now for saving lives. Law Contemp. Probl. 40, 5-45 (1976).

34. Kauffman, A. L., Ashraf, J. M., Corces-Zimmerman, M. R., Landis, J. N. \& Murphy, C. T. Insulin signaling and dietary restriction differentially influence the decline of learning and memory with age. PLoS Biol. 8, e1000372 (2010).

35. Evans, E. A., Kawli, T. \& Tan, M. W. Pseudomonas aeruginosa suppresses host immunity by activating the DAF-2 insulin-like signaling pathway in Caenorhabditis elegans. PLoS Pathog. 4, e1000175 (2008).

36. Cohen, E., Bieschke, J., Perciavalle, R. M., Kelly, J. W. \& Dillin, A. Opposing activities protect against age-onset proteotoxicity. Science 313, 1604-1610 (2006).

37. Mulcahy, B., Holden-Dye, L. \& O'Connor, V. Pharmacological assays reveal age-related changes in synaptic transmission at the Caenorhabditis elegans neuromuscular junction that are modified by reduced insulin signalling. J. Exp. Biol. 216, 492-501 (2013).

38. Luo, S., Kleemann, G. A., Ashraf, J. M., Shaw, W. M. \& Murphy, C. T. TGF-beta and insulin signaling regulate reproductive aging via oocyte and germline quality maintenance. Cell 143, 299-312 (2010).

39. Yang, J. S. et al. OASIS: online application for the survival analysis of lifespan assays performed in aging research. PLoS ONE 6, 11 (2011).

\section{Acknowledgements}

We thank the Caenorhabditis Genetics Center for strains. We are grateful to Professor Jan Vijg, Professor Yousin Suh and Professor Kyuhyung Kim for their valuable suggestions. This work was supported by the Research Center Program of the Institute for Basic Science (project code: IBS-R013-D1) and the National Institutes of Health (Grant number 5T32GM007388-38). CTM is the Director of the Glenn Center for Aging Research at Princeton University. R.D. is supported by NIH 5T32GM007388-38, and C.S. by March of Dimes.

\section{Author contributions}

J.H.H., C.T.M., and H.G.N. conceived and designed the study and wrote the manuscript, J.H.H., S.K., R.D., and C.S. performed the experimental works and analyzed the data, S.K. performed MV and pumping assays (Fig. 2 and Supplementary Fig. 5), R.D. did the quality analyses (Fig. 4 and Supplementary Fig. 11), C.S. performed the on-bacteria motility assays (Fig. 3 and Supplementary Fig. 10), J.H.H., S.J.L., C.T.M., and H.G.N edited the manuscript.

\section{Additional information}

Supplementary Information accompanies this paper at http://www.nature.com/ naturecommunications

Competing financial interests: The authors declare no competing financial interests.

Reprints and permission information is available online at http://npg.nature.com/ reprintsandpermissions/

How to cite this article: Hahm, J.-H. et al. C. elegans maximum velocity correlates with healthspan and is maintained in worms with an insulin receptor mutation. Nat. Commun. 6:8919 doi: 10.1038/ncomms9919 (2015).

This work is licensed under a Creative Commons Attribution 4.0 International License. The images or other third party material in this article are included in the article's Creative Commons license, unless indicated otherwise in the credit line; if the material is not included under the Creative Commons license, users will need to obtain permission from the license holder to reproduce the material. To view a copy of this license, visit http://creativecommons.org/licenses/by/4.0/ 